\title{
Vergleichende Histologie des Nackenbandes.
}

\author{
Von \\ Hidetosi Ohara. \\ (Aus dem Anatomischen Institut der Medizinischen Fakultät zu \\ Okayama ; Vorstand : Prof. M. Scki u. Prof. R. Ura.) \\ Mit 6 Abbildungen auf Tafel XXIX.
}

Auf Grund histologischer Untersuchungen äußerte sich Ka to (1939) bezüglich des menschlichen Nackenbandes dahin, daB das in der Jenaer Nomina anatomica als Septum nuchae bezeichnete Nackenband in der Wirklichkeit wie bisher Ligamentum nuchae genannt werden muß. Angesichts der Tatsache, daß sich das Nackenband auch beim anfrecht gehenden Menschen ziemlich stark entwickelt, drängte sich nun die Frage auf, ob und wieweit bei verschiedenen Tieren, welche in der Größe des Kopfes, in der 'Länge des Halses und im Habitus voneinander sehr abweichen, das Nackenband morphologisch und histologisch Unterschiede aufweist.

\section{Material und Methode.}

Als Material dienten folgende Tiere: r. Reh aus Korea (Capreolus bedfordi), 2. Reh aus Formosa, Kiyon (Muntiacus reevesii micrurus), 3. japanischer Hirsch (Cervus nippon), 4. Kängutuh (Macropus giganteus), s. malaiischer Bär (Ursus thibetanus malayanus), Dachs (Nyctereutes viverrinus), 7. krebsfressende Manguste (Herpstes urva), 8. Seidenaffe (Hapale jaccbus).

All diese Tiere wurden in einem Tiergarten gehalten und starben daselbst. Nach der Fixierung des Tierkörpers durch die Injektion von Formalinlösung in die Blutgefäße wurde der mittlere Teil des Nackenbandes samt den benachbarten Anteilen der Muskulatur entnommen und in Zelloidin eingebettet, um Querschnitte von $30 \mu$. Dicke anzufertigen und diese mit Hämatoxylin und Eosin und mit Resorzin-Fuchsin zu färben:

\section{Befunde der Untersuchung.}

Das Nackenband des Menschen läßt sich, worauf Ka to aufmerksam 
gemacht hat, in zwei Teile, in einen dorsalen dickeren Bandteil(Nackenstrang) und in einen membranartigen Septalteil (Nackenplatte) einteilen. Bei allen von mir untersuchten Tieren bestand das Band mehr oder weniger deutlich aus solchen $z$ wei Teilen.

I. Reh aus Korea $(20,5 \mathrm{~kg})$.

Es hat das stärkste Nackenband uhter den von mir untersuchten Tieren. Der Bandteil, der im Querschnitt eine querovale Form darbietet, beträgt etwa $12 \mathrm{~mm}$ im Querdurchmesser und etwa $7 \mathrm{~mm}$ im dorsoventralen Durchmesser (Abb. I). Dieser Anteil des Nackenbandes besteht, das mediane bindegewebige Septum ausgenommen, aus dicht gedrängten dicken elastischen Fasern (Abb. 4). Das interstitielle Bindegewebe ist sehr spärlich vothanden. Der Septalteil teilt sich in zwei Blätter, welche bald quer, bald längslaufende dicke elastische Fasem in reichlicher Menge in sich einschließen.

2. Reh aus Formosa, Kiyon $(9,5 \mathrm{~kg}$ ).

Auch bei diesem Tiere entwickelt sich der Bandteil des Nackenbandes sehr stark. Sein Querdurchinesser beträgt etwa $\times 2 \mathrm{~mm}$, der dorsoventrale Durchmesser etwa $4 \mathrm{~mm}$. Der Querschnitt ähnelt einem Regenschirm, der Septalteil stellt dabei den Handgriff des Schirmes dar (Abb. 2). Der mikroskopische Bau des Bandes ist der gleiche wie beim Reh aus Korea, nut daß das interstitielle Bindegewebe zwischen den elastischen Fasern etwas. ärmer ist.

3. Japanischer Hirsch ( $26 \mathrm{~kg})$.

Hier entwickelt sich das Nackenband wieder sehr gut. Die Querschnittsform ist aber anders als bei den obigen. Der Bandteil beträgt nämlich etwa $4 \mathrm{~mm}$ im Querdurchmesser und etwa $10 \mathrm{~mm}$ im dorsoventralen Durchmesser, sein Querschnitt sieht wie eine Keule aus (Abb. 3). Das lockere Bindegewebe dringt, Blutgefäße begleitend, von der Oberfläche in ihn hinein, ohne daß es die elastischen Fasern deutlich in Bündel einteilt. Der Septalteil ist zwar dünn, enthält aber auch reichlich lāngs- und schräglaufende elastische Fasern:

4. Känguruk (II kg).

Das Nackenband des Känguruhs entwickelt sich im Vergleich zu den obigen viel schlechter. Der Bandteil besteht wie bei den gewöhnlichen Sehnen aus parallelen dicken kollagenenfasem, welehe durch lockeres Bindegewebe zu Bündeln verchiedener Größe vereinigt sind. Die elastischen Fasem sind nur in dem $z$ wischen solchen Bündeln gelagerten Bindegewebe zu sehen, sie sind fein und spärlich (Abb. s). Der Septalteil ist dünn und enthält wenige längslaufende, vethältnismäßig dicke elastische Fasern.

5. Malai ischer Bär $(25,5 \mathrm{~kg})$.

Der Bandteil des Nackenbandes, etwa $3 \mathrm{~mm}$ im Quer- und etwa 4,5 $\mathrm{mm}$ 
im dorsoventralen Durchmesser, ist in Anbetracht der Körpergröße verhältnismäßig schwach. Sein Bau ist fast der gleichē wie beim Känguruh. Die elastischen Fasern sind im Septalteil arm.

6. Dachs ( $5 \mathrm{~kg})$.

Beim Dachs ist das Nackenband nur schwach entwickelt. Der Bandteil ist von etwa 0,4 $\mathrm{mm}$ Querdurchmesser, der Septalteil aber von etwa 0,2 mm. Der Bandteil schickt auf beiden Seiten je 2-3 kuze Fortsätze zwischen die Muskelgruppen ein. Die elastischen Fasern sind im allgemeinen spärlich zu finden.

7. Krebsfressende Manguste (2,6 kg).

Hier ist auch der Bandteil des Nackenbandes auf beiden Seiten mit 2-3 kurzen Fortsätzen versehen. Die elastischen Fasern sind in der oberflächlichen Schicht des Bandteils spärlich und im Septalteil viel zu sehen. Die Fasem im letzteren sind übrigens dick und meistens schräglaufend. Die Manguste ist im Körpergewicht nur halb so groß wie beim Dachs. Der Bandteil des Nackenbandes ist aber sowohl in der Breite als auch in der Länge etwa doppelt so groß wie bei ihm.

8. Seidenaffe $(0,25 \mathrm{~kg})$.

Der Seidenaffe erreicht im Gewicht kaum I/ro der Manguste, übertrifft aber sie in der Stärke des Nackenbandes. Die elastischen Fasern sind sehr spärlich. Genauers in der nachstehenden Tabelle.

\section{Besprechung.}

Das Nackenband der von mir untersuchten Tiere läßt sich wie beim Menschen in den Band- und Septalteil einteilen. Beim Reh aus Korea und Formosa sowie beim japanischen Hirsch, welche alle einen lang vorgestreckten Hals besitzen und ihn häufig nach oben und unten bewegen, ist das Nacken-

\begin{tabular}{|c|c|c|c|c|c|}
\hline & \multirow{2}{*}{$\begin{array}{l}\text { Körper- } \\
\text { gewicbbt } \\
\text { (kg) }\end{array}$} & \multicolumn{2}{|c|}{ Breite des Bandteils - " } & \multicolumn{2}{|c|}{-Elastische Fasern } \\
\hline - & & $\begin{array}{l}\text { Quer- } \\
\text { dutchmesser } \\
\text { (mm) }\end{array}$ & $\begin{array}{c}\text { Dorsoveats. } \\
\text { Durchmessex } \\
\text { (mm) }\end{array}$ & $\begin{array}{c}\text { in } \\
\text { Bandteil }\end{array}$ & $\begin{array}{l}\text { in } \\
\text { Septalteil }\end{array}$ \\
\hline Reh aus Korea & 20,5 & 12 & 7 & $+t+$ & ++ \\
\hline Reh aus Formosa, Kiyon & $: 9,5$ & 12 & 4 & $++t$ & ++ \\
\hline Japanischer Hirsch & 26 & .4 & 10 & +++ & ++ \\
\hline Kănguruh & $x x^{\circ}$ & $\mathbf{I}$ & 0,8 & +++ & + \\
\hline Malaiischer Băr & 25,5 & 3 & 4,5 & \pm & \pm \\
\hline Dachs & 5 & 0,4 & 0,8 & \pm & \pm \\
\hline Krebsfressende Manguste & 2,6 & 0,7 & $x, 5$ & \pm & + \\
\hline Seidenaffe & 0,25 & 0,8 & 1,5 & \pm & \pm \\
\hline
\end{tabular}


band besonders stark entwickelt. Der Bandteil besteht fast rein aus elastischen Fasern. Auch der Septalteil ist an elastischen Fasem sehr reich. Die Stärke des Nackenbandes ist übrigens wie aus der Tabelle zu ersehen ist, dem Körpergewicht keineswegs proportional. Beim großen malaiischen Bär z. B. ist die Entwicklung des Nackenbandes nur schlecht, die elastischen Fasern sind in ihm sehr arm.

Bei der krebsfressenden Manguste wird die wenige Vertikalbewegung seines langen Halses wohl dafür die Ursache sein, daß̣ sein verhältnismäßig starkes Nackenband an elastischen Elementen arm ist.

Beim Känguruh ist das Nackenband nicht stark entwickelt. Der Tier hat ein verhältnismäßig kleines Haupt und hält gewöhnlich den Hals aufrecht. Der Band ist übrigens mit lockerem Bindegewebe stark durchsetzt. Da mmann (r9a8) verglich verschiedene Sehnen von Rind und Pferd und bestätigte, daß das Peritenonium internum beim Rind viel stärker entwickelt ist als beim Pferd. Er sah ferner, daß die Sehnen von verhältnismäßig schwächeren Muskeln das lockere Bindegewebe in viel reichlicherer Menge enthält als diejenigen von stärkeren Muskeln.

Der Seidenaffe besitzt ein verhältnismäßig starkes Nackenband, was wohl auf seinen verhältnismäBig schweren Kopf zu beziehen ist. Das Nackenband ist aber arm in der Menge der elàstischen Fasern.

\section{Zusammenfassung.}

1. Bei der Familie des Hirsches, welche einen langen Hals häufig nach oben und unten bewegt, entwickelt sich das Nackenband besonders gut. Sein dorsaler, stark verdickter Bandteil ist fast rein elastisch. Der dünnere Septalteil ist reichlich mit elastischen Fasern versehen.

2. Bei den Tieren mit einem kürzeren Hals (malaiischer Bär und Dachs) besteht der Bandteil des Nackenbandes hauptsächlich aus dicken kollagenen Fasem. Die elastischen Elemente sind aber nur schwach entwickelt. Histologisch gehört hierzu das Nackenband des Känguruhs, welches zwar einen langen Hals besitzt, gewöhnlich aber dies aufrecht hālt.

3. Die krebsfressende Manguste mit einem lang vorgestreckten, aber wenig vertikalbewegenden Hals hat das verhältnismäßig starke, aber wenig elastische Nackenband. Der Seidenaffe hat ein verhältnismäßig großes Haupt. Sein Nackenband ist strukturiert wie bei der Manguste.

\section{Literaturverzeichnis.}

Damman n, O.: Vërgleichènde Untersuchungen über den Bau und die funktionelle Aapassung der Sehnen. Arch. Entw. 26 (1908).

Ellenberget, W. u. Baum, H. : Vergleichende Anatomie der Haustiere. Berlin r926. 
Fick,'R.: Anatomie und Mechanik der Gelenke. In Bardelebens Handbuch der Anatomie des Menschen, Bd. 2, I. Abt., x. Teil. Jana I904.

Kato, T.: Histologische Untersuchungen über das Nackenband des Menschen. Arb. Med. Fak. Okayama. 6 (1939).

\section{Erklärung der Abbildungen.}

Abb. t. Querschnitt des Bandteils des Nackenbandes des Rehs aus Korea in det mittleren Höhe. Resorzin-Fuchsinfärbung. Vergr. $5 \times$.

Abb. 2. Vom Reh aus Formosa, Kiyon. Beschreibung sonst wie oben.'

Abb. 3. Vom japanischen Hirsch. Beschreibung wie oben.

Abb. 4. Aus dern Bandteil des Nakenbandes des Rehs aus Korea. Vergr 70X.

Abb. 5. Vom Kangquruh. Beschreibung sonst wie oben.

Abb. 6. Bandteil des Nackenbandes der, krebsfressenden Manguste. Hămatoxylin-Eosinfätbung. Vergr. $35 \times$. 

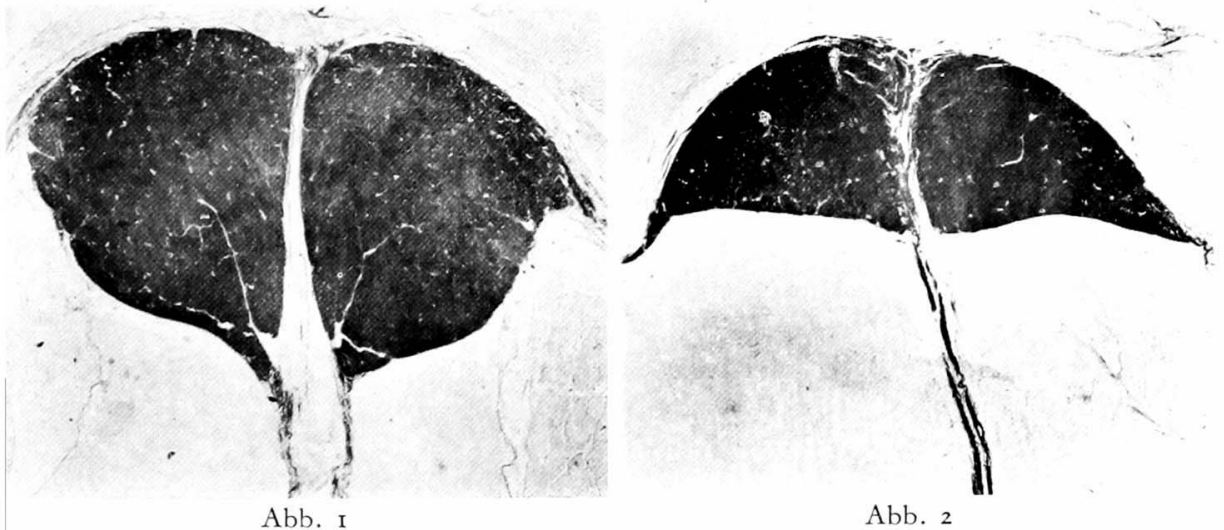

Abb. 2
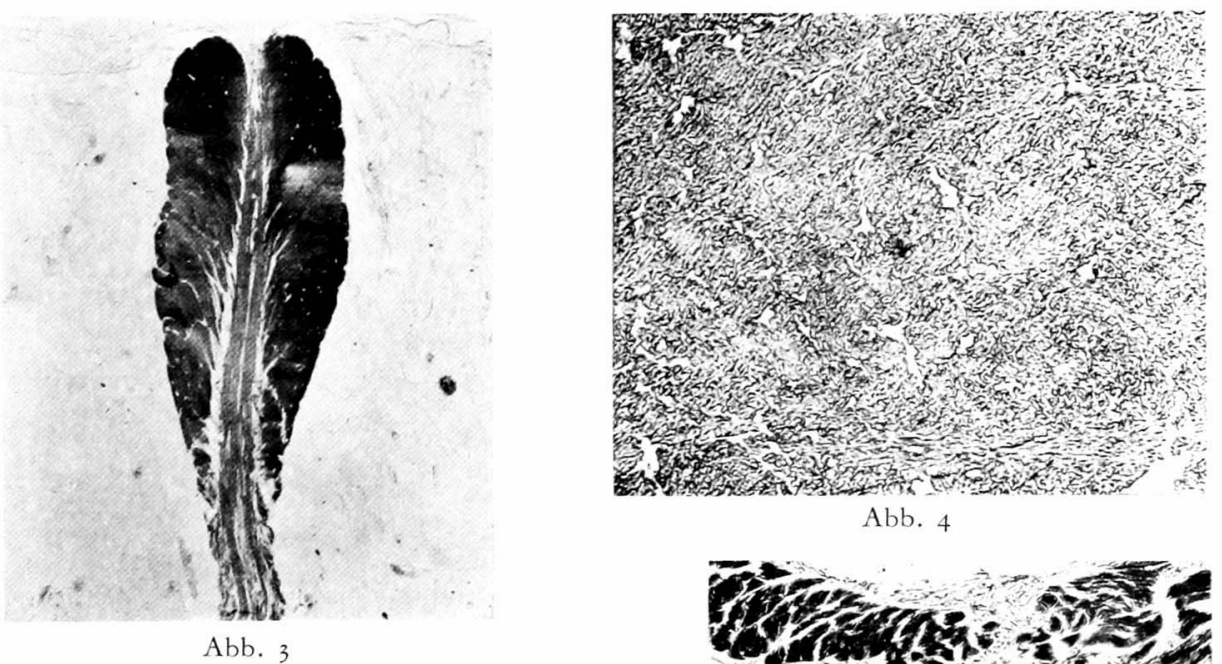
Abb. 4
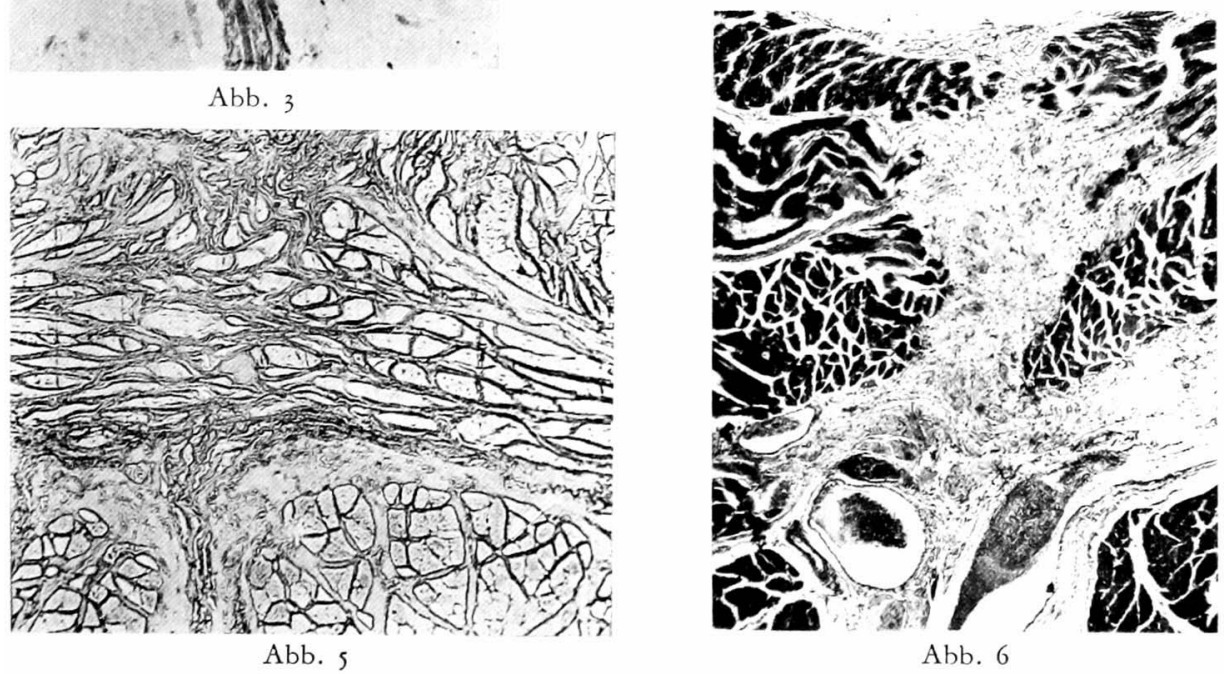
Abb. 6

H. Ohara 